\title{
РЕГРЕССИОННОЕ МОДЕЛИРОВАНИЕ В ЗАДАЧАХ ЭКОНОМИКИ ТРУДА
}

\section{REGRESSION MODELING IN THE TASKS OF LABOR ECONOMICS \\ D. Aidinova}

Summary. The article studies the effectiveness of the method of correlation-regression and economic-mathematical modeling for solving poorly studied, but the most common problems in the labor economy:

modeling of the relationship between labor productivity and work experience;

- determination of the optimal time-consuming option;

- optimal distribution of workers by type of work;

- determination of effective production rates and time standards;

- study of the use of working hours and the operation of equipment.

Keywords: labor, labor price, labor productivity, work experience, employment, unemployment, types of connections, modeling of connections, tightness of communication, optimal time costs, regression model, regression coefficient, correlation coefficient, regression equation.

$\mathbf{K}$ ак известно, регрессионный анализ в настоящее время находит широкое применение в микроэкономическом анализе. Регрессионные модели сегодня все чаще применяются также и в макроэкономике как инструмент количественного анализа связи между социально-экономическими явлениями и их признаками. Путём построения регрессионных моделей становится возможным выражение зависимостей между исследуемыми факторами, которые основаны на специальной обработке статистических данных за длительный период времени.

В прикладной литературе часто употребляется в одном и том же смысле корреляционный и регрессионный анализ. В целях уяснения терминологии сначала уточним разницу между ними.

При построении регрессионных моделей как правило, исходят из того, что зависимая величина Ү являет-

\author{
Айдинова Диана Хаджи-Муратовна \\ К.э.н., доцент кафедры экономики и прикладной \\ информатики, Карачаево-Черкесский \\ государственный университет имени У.Д. Алиева, \\ 2. Карачаевск, Россия \\ diana.aidinova@mail.ru
}

Аннотация. В статье проводится исследование эффективности действия метода корреляционно-регрессионного и экономико-математического моделирования для решения малоизученных, но наиболее часто встречающихся проблем в экономике труда:.

- моделирование связи производительности труда и стажа работы;

- определение оптимального варианта затрат времени;

- оптимальное распределение рабочих по видам работ;

- определение эффективных норм выработки и норм времени;

- изучение использования рабочего времени и работы оборудования.

Ключевые слова: труд, цена труда, производительность труда, стаж работы, занятость, безработица, виды связей, моделирование связей, теснота связи, оптимальные затраты времени, регрессионная модель, коэффициент регрессии, коэффициент корреляции, уравнение регрессии. ся случайной величиной, а её распределение зависит от конкретных значений одной или нескольких зависимых переменных $\mathrm{X}_{1}, \mathrm{X}_{2}, \ldots, \mathrm{X}_{n}$, которые рассматриваются как неслучайные.

Следует отметить, что в предстоящем нашем исследовании регрессионному анализу всегда будет предшествовать корреляционный анализ, где связь между величинами $\mathrm{X}_{1}, \mathrm{X}_{2}, \ldots, \mathrm{X}_{n}$ описывается в виде многомерного распределения, а сами величины здесь являются случайными величинами. Следует отметить, что корреляционный анализ почти всегда дополняется регрессионным анализом. Напомним, что при этом регрессионный анализ позволяет изучить изменение средней величины $\mathrm{Y}$ как функцию от случайных величин $\mathrm{X}_{1}, \mathrm{X}_{2}$, $\ldots, \mathrm{X}_{n} .[2$, c. 18$]$.

Целью нашего исследования является привлечение метода корреляционно-регрессионного анализа к из- 
$y$, urr.

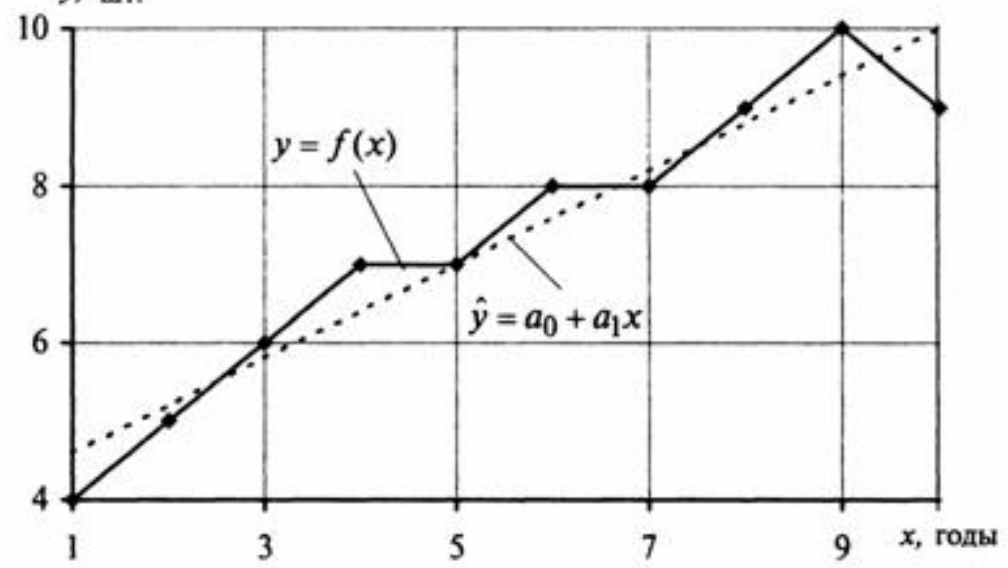

Рис. 1. Зависимость выработки одного рабочего Ү от стажа работы X

учению тех малоизученных социально-экономических явлений, когда статистическая зависимость, как правило, не соответствовала причинам связи или когда статистическая независимость скрывала причинную связь. При этом особое внимание обращается обоснованию выбора факторов, влияющих на результат.

Одной из наиболее часто применяемых в экономических исследованиях моделей является модель парной линейной связи. При этом регрессионное моделирование задач экономики труда является одним из малоизученных направлений экономического анализа. На наш взгляд, исследование проблем экономики труда следует начинать с производительности труда, т.к. она является ключевым исходным фактором, влияющим на эффективность производства продукции предприятия.

На наш взгляд, исследование необходимо провести в следующей последовательности:

1. Сначала необходимо, используя данные таблицы 1, построить однофакторное уравнение регрессии зависимости производительности труда Ү от стажа работы X.

При проведении исследования, исходя из экономических соображений, стаж работы нами выбран в качестве независимой переменной Х. Сопоставление данных признаков $X$ и $Y$ достаточно ясно показывает, что с возрастанием стажа работы производительность труда растет, хотя и не всегда. Следовательно, между $\mathrm{X}$ и Y, на наш взгляд, скорее всего, существует прямая зависимость.

2. Для уточнения формы связи между рассматриваемыми признаками используем графический метод ана- лиза. Для этого нанесем на график точки, соответствующие значениям X и Ү. Соединив их отрезками, получим ломаную линию регрессии (рис. 1). Анализируя ломаную линию, можно предположить, что возрастание производительности труда рабочихҮ идет равномерно и пропорционально росту стажа работы Х. В основе этой зависимости лежит прямолинейная связь, которая может быть выражена простым линейным уравнением регрессии:

$$
\hat{y}=a_{0}+a_{1} x
$$

Для удобства все расчеты проведены нами в таблице 1 , где $\hat{y}_{i}$ - теоретические расчетные значения производительности труда одного рабочего (шт.), полученные по уравнению регрессии; $a_{0}, a_{1}$ - неизвестные параметры уравнения регрессии; $x$ - стаж работы рабочих (год).

3. Пользуясь расчетными значениями показателей (табл. 1), исчислим параметры для составления регрессионной модели:

$$
a_{1}=\frac{\overline{x y}-\overline{x y}}{\overline{x^{2}}-x^{-2}}=\frac{45,1-40,15}{38,5-30,25} \approx 0,6
$$

Следовательно, регрессионная модель распределения выработки рабочих по стажу работы для данного случая может быть записана в виде простого уравнения регрессии: $y=4,0+0,6 x$. Это уравнение характеризует зависимость среднего уровня выработки рабочих бригады от стажа работы:

при росте стажа работы на 1 год выработка рабочего повысится в среднем на 0,6 ед. [4, с. 15].

Следует отметить, что для практического использования моделей регрессии ключевое значение имеет 
Таблица 1. Распределение рабочих бригады по выработке и стажу работы

\begin{tabular}{|c|c|c|c|c|c|c|}
\hline \multicolumn{3}{|c|}{ Исходные данные } & \multicolumn{4}{|c|}{ Расчетные значения } \\
\hline $\begin{array}{l}\text { Номер } \\
\text { рабочего }\end{array}$ & $\begin{array}{l}\text { Стаж работы, } \\
\text { годы } x\end{array}$ & \begin{tabular}{|l} 
Дневная \\
выработка, шт. у
\end{tabular} & $x^{2}$ & $y^{2}$ & $x y$ & $\hat{y}_{i}$ \\
\hline $4-\breve{~}$ & 1 & 4 & 1 & 16 & 4 & 4,6 \\
\hline $6-и ̆$ & 2 & 5 & 4 & 25 & 10 & 5,2 \\
\hline $3-и ̆$ & 3 & 6 & 9 & 36 & 18 & 5,8 \\
\hline $1-и ̆$ & 4 & 7 & 16 & 49 & 28 & 6,4 \\
\hline $2-\breve{n}$ & 5 & 7 & 25 & 49 & 35 & 7,0 \\
\hline $7-и ̆$ & 6 & 8 & 36 & 64 & 48 & 7,6 \\
\hline $9-и ̆$ & 7 & 8 & 49 & 64 & 56 & 8,2 \\
\hline 10-й & 8 & 9 & 64 & 81 & 72 & 8,8 \\
\hline $8-и ̆$ & 9 & 10 & 81 & 100 & 90 & 9,4 \\
\hline $5-и ̆$ & 10 & 9 & 100 & 81 & 90 & 10,0 \\
\hline Итого & $\Sigma x=55$ & $\Sigma y=73$ & $\Sigma x^{2}=385$ & $\Sigma y^{2}=565$ & $\Sigma x y=451$ & 73,0 \\
\hline
\end{tabular}

максимальное соответствие моделей фактическим статистическим данным. Как известно, на предприятиях малого и среднего бизнеса корреляционно-регрессионный анализ обычно проводится для небольшого количества единиц совокупности. В этом случае показатели регрессии и корреляции могут быть искажены под действием случайных факторов. При этом появляется необходимость проверить, насколько эти показатели характерны для всей большой совокупности, не являются ли они результатом стечения случайных обстоятельств.

4. Для проверки значимости коэффициентов регрессии исследуемого уравнения $y=4,0+0,6 x$ необходимо вычислить $t$-критерий Стьюдента с $v=10-2=8$ степенями свободы. Рассчитаем также среднее квадратическое отклонение:

$$
\begin{aligned}
& \sigma_{\text {ocm }}=\sqrt{\frac{\sum(\hat{y}-y)^{2}}{n}}=\sqrt{\frac{2,4}{10}}=0,49, \\
& \sigma_{x}=\sqrt{\frac{\sum x^{2}}{n}-\left(\frac{\sum x}{n}\right)^{2}}=\sqrt{\frac{385}{10}-\left(\frac{55}{10}\right)^{2}}=2,87
\end{aligned}
$$

Тогда расчетные значения $t$-критерия Стьюдента составят:

$$
t_{a_{0}}=4 \frac{\sqrt{10-2}}{0,49}=23,1, t_{a_{1}}=0,6 \frac{\sqrt{10-2}}{0,49} \cdot 2,87=9,94
$$

Для $v=8$ находим критическое значение $t$-критерия: $\left(t_{\text {табл }}=3,307\right.$ при $\left.\mathrm{a}=0,05\right)$. Поскольку расчетное значение $t_{\text {расч }}>t_{\text {табл }}$, оба параметра $a_{0}, a_{1}$ признаются значимыми.

5. Оценим тесноту связи между стажем рабочих и производительностью труда.
Как известно, при использовании линейной формы уравнения для оценки тесноты связи часто применяется линейный коэффициент корреляции:

$$
r=\frac{451-401,5}{\sqrt{82,5 \cdot 32,1}}=\frac{49,5}{51,46} \approx 0,962
$$

Используя данные табл. 1, рассчитаем линейный коэффициент корреляции Коэффициент детерминации $\mathrm{r}^{2}$ равен 0,925 , свидетельствует о наличии тесной прямой зависимости между рассматриваемыми признаками $\mathrm{X}$ и Ү. Следовательно, с вероятностью 0,95 можно утверждать, что 92,5\% общей вариации производительности труда рабочих бригады объясняется вариацией стажа работы, а 7,5\% объясняется действием других факторов.

Используя для оценки значимости коэффициента корреляции между производительностью труда и стажем работы $t$-критерий Стьюдента, получим:

$$
t_{\text {pacu }}=0,961 \sqrt{\frac{10-2}{1-0,925}} \approx 9,93
$$

Это значительно больше критического значения $t$ для $n-2=8$ степеней свободы и $а=0,01\left(t_{\text {табл }}=3,356\right)$, что свидетельствует о значимости коэффициента корреляции и существенности связи между производительностью труда рабочих бригады и стажем их работы.

Таким образом, построенная нами регрессионная модель $\hat{y}=4,0+0,6 x$ в целом является верной и точно соответствует фактическим статистическим данным. Выводы, полученные по результатам данной малой выборки, с достаточно высокой вероятностью 0,95 можно распространить на всю генеральную совокупность. [5, c. 37]. 
Таблица 2

\begin{tabular}{|l|l|l|l|}
\hline База & $X_{0}$ & $X_{1}$ & $X_{2}$ \\
\hline$y_{1}$ & 8 & 1 & 2 \\
\hline$y_{2}$ & 7 & 2 & 1 \\
\hline$y_{3}$ & 6 & 1 & 1 \\
\hline$C$ & 0 & -4 & -4 \\
\hline
\end{tabular}

Следующей малоизученной проблемой экономики труда является изучение затрат рабочего времени в практике технического нормирования. Изучение затрат рабочего времени осуществляется путем непосредственного наблюдения методом хронометража и фотографии рабочего дня. Решение подобной задачи мы предлагаем провести обычным симплекс-методом. Предлагается исследовать несколько однородных рабочих мест, специализированных на выполнении операций двух видов. На каждом рабочем месте за один и тот же календарный период выполняются все или часть операций. При этом под фондом времени понимается все отработанное, а не только полезное время. Формула, связывающая фонд времени и затраты на выполнение отдельных операций, имеет вид: $\Phi \geq a_{1} \bar{X}_{1}=a_{2} \bar{x}_{2}=\ldots=a_{n} \bar{x}_{n}$, где $a_{1}, a_{2}, \ldots, a_{n}-$ число отдельных операций, выполненных за данный период: $\bar{X}_{1} \cdot \bar{X}_{2}, \ldots \bar{X}_{n}-$ искомые средние затраты времени на каждую операцию.

Особенности решения этой задачи сначала рассмотрим на несколько упрощенном примере. Предположим, конструктор за первые 8 дней работы сконструировал одну деталь первой группы сложности и две - второй группы; в следующие 7 дней он сконструировал две детали первой группы сложности и одну - второй, а в последние 6 дней одну деталь первой и одну - второй группы сложности. Эти условия задачи могут быть записаны в виде неравенств:

$$
\left\{\begin{array}{l}
x_{1}+2 x_{2} \leq 8 \\
2 x_{1}+x \leq 7 \\
x_{1}+x_{2} \leq 6
\end{array}\right.
$$

где $X_{1}$ - время конструирования одной детали первой группы сложности; $X_{2}$ - время конструирования одной детали второй группы сложности. Запись времени на конструирование деталей этих групп сложности в виде неравенств показывает, что конструктор мог иметь потери времени за счет различных непроизводительных затрат труда. Обозначив потери времени в течение трех периодов работы соответственно через $y_{1}, y_{1}, y_{3} ;$ перейдем к системе уравнений:

$$
\begin{aligned}
& x_{1}+2 x_{2}+y_{1}=8, \\
& 2 x_{1}+x_{2}+y_{2}=7, \\
& x_{1}+x_{2}+y_{3}=6
\end{aligned}
$$

Далее определяется линейная функция, которая минимизирует суммарные затраты времени, представленные дополнительными переменными $\left(y_{1}, y_{1}, y_{3}\right)$. Эта функция является суммой всех уравнений (2):

$$
4 x_{1}+4 x_{2}+Q=21,
$$

где $Q=y_{1}+y_{2}+y_{3}-$ сумма дополнительных переменных (или непроизводительных затрат времени). Из уравнения (3) видно, что за 21 рабочий день конструктор сконструировал 8 деталей: 4 детали первой группы сложности и 4детали второй группы (в общий срок работы включаются и непроизводительные затраты).

Задача заключается в том, чтобы найти такие значения $X_{1}$ и $X_{2}$, которые бы сводили к минимуму сумму непроизводительных затрат $\left(y_{1}+y_{2}+y_{3}-\min \right)$, или, что и одно и тоже, максимально приблизили бы время производительной работы к 21 дню ( $\left.4 x_{1}+4 x_{2}=\max \right)$. Может быть избран любой критерий в зависимости от условий конкретной задачи. Решается задача симплексным методом. Для этого необходимо найти по крайней мере один вариант, не противоречащий системе (2), либо принять вариант с нулевыми затратами на основные работы: $x_{1}=0$ и $x_{2}=0$. В таком случае потери и непроизводительные затраты рабочего времени будут равны величине, стоящей в правой части системы (2): $y_{1}=8, y_{2}=7, y_{3}=6$. Этот вариант также не противоречит системе (2).

Исходя из нулевого варианта, путем последовательного изменения значений $X_{1}$ и $X_{2}$ следует добиться максимально возможного значения суммы $4 X_{1}+4 x_{2}$.

С этой целью строится первая симплексная таблица. Исходные данные записываются в виде системы уравнений: 


$$
\begin{aligned}
& 8=x_{1}+2 x_{2}+y_{1} \\
& 7=2 x_{1}+x_{2}+y_{2} \\
& 6=x_{1}+x_{2}+y_{3}=6 \\
& 0-C-4 x_{1}-4 x_{2}
\end{aligned}
$$

И в табличной форме (таблица 2).

После проведения трех итераций по алгоритму симплекс-метода на последней симплексной таблице получим оптимальный вариант затрат времени:

$$
\begin{array}{cc}
x_{1}=2 ; & y_{1}=0, \\
x_{2}=3 ; & y_{2}=0, \\
y_{3}=1
\end{array}
$$

Это означает, что на конструирование одной детали первой группы сложности тратиться 2 дня, а второй 3 дня. В первые два периода работы непроизводительных затрат времени не было, а в третий период имел место простой в течение одного дня.

Многие задачи экономики, в том числе задачи оптимизации организации и нормирования труда (например, оптимальное распределение рабочих на конвейере и на поточной линии, распределение рабочих по видам работ и т.д.) можно решить методом динамического программирования, где учитываются временные изменения и используется аппарат рекуррентных соотношений [3].

Процесс решения задачи расчленяется на этапы, которые решаются последовательно во времени, а также на этапы, которые, в конечном счете, приводят к искомому решению.

Рассмотрим сущность метода динамического программирования при решении задачи распределения рабочих по видам работ и рабочим местам.

Экономическая постановка задачи. Имеется некоторое количество работ с различной производительностью. Требуется так распределить имеющихся в наличии рабочих, чтобы получить максимальную производительность по всей совокупности работ. При этом количество производимой продукции может зависеть от количества рабочих нелинейно. В качестве критерия оптимальности используется показатель производительности труда по всей совокупности работ. Требование достичь максимальной производительности при постоянной численности рабочих означает повышение объема продукции при той же численности рабочих.

Для математической постановки задачи введем обозначения:

$X$ - общая численность рабочих;

$i$ - номер трудовых процессов $(i=1,2, \ldots, v)$;
$X_{i}$ - численность рабочих, выделенных для выполнения $i$-го вида работ;

$Y_{i}$ - количество продукции на $i$-ом виде работ;

$Y$ - общий выпуск продукции по всем видам работ (процессам).

В этих обозначениях условие 3 запишется так:

$$
Y\left(x_{1}, x_{2}, \ldots, x_{n}\right)=y_{1}\left(x_{1}\right)+y_{2}\left(x_{2}\right)+\ldots .+y_{n}\left(x_{n}\right)
$$

Кроме того выполняются условия:

а) численность рабочих не может быть отрицательной $x_{i} \geq 0 \quad(i=1,2, \ldots, n)$;

б) общая численность рабочих полностью распределяется по всем процессам

$$
\sum_{i=1}^{n} X_{i}=X
$$

Исходя из принципа вложения, можем рассматривать задачу в общем виде, то есть считать, что $X_{n} n$ принимают любые положительные значения.

Введем функцию

$$
\begin{aligned}
& f_{n}(x)=\max Y\left(x_{1}, x_{2}, \ldots, x_{n}\right) \\
& x_{i} \geq 0 \quad(i=1,2, \ldots, n) \\
& \sum_{i=1}^{n} x_{i}=X
\end{aligned}
$$

Функция $f_{n}(X)$ выражает максимальное значение функции $Y\left(x_{1}, x_{2}, \ldots, x_{n}\right)$ при оптимально выбранных $x_{1}, x_{2}, x_{3}, \ldots, x_{n}$ т.е. максимальную производительность труда при оптимальном распределении $X$ рабочих по $n$ трудовым процессам.

Функция $f_{n-1}(X-x)$ обозначает максимальный выпуск продукции, полученный при распределении $\left(X-X_{n}\right)$ рабочих, по $(n-1)$ процессу.

Положим $y_{i}(0)=0 \quad(i=1,2, \ldots, n)$, что вполне возможно в заданной задаче. Тогда $f_{n}(0)=0$ для любой $i \quad(i=1,2, \ldots, n)$, так как $f_{n}(0)=\sum_{i=1}^{n} Y_{i}(0)$

Если $X \geq 0$, то $f_{1}(X)=y_{1}(X)$, то есть если всех рабочих отдать в первый процесс, то максимальный выпуск продукции, учитывая, что при $X_{1}=X$ все $X_{2}=X_{3}=\ldots .=X_{n}=0$ и $y_{i}(0)=0 \quad(i=1,2, \ldots, n)$, будет равен выпуску продукции в первом процессе.

Чтобы придать статическому процессу динамический характер, решение задачи проводится по этапам. 
Таблица 3

\begin{tabular}{|l|l|l|l|l|l|l|}
\multicolumn{1}{l|}{$f_{l}(x)$} & $f_{2}(x)$ & $f_{3}(x)$ & $f_{4}(x)$ & $\ldots$ & $f_{n}(x)$ \\
\hline 0 & & & & & & \\
\hline$\Delta$ & & & & & & \\
\hline $2 \Delta$ & & & & & & \\
\hline$\ldots$ & $\ldots$ & $\ldots$ & $\ldots$ & $\ldots$ & $\ldots$ & $\ldots$ \\
\hline$X$ & & & & & & \\
\hline
\end{tabular}

На первом этапе выделяется какое-то количество рабочих $i$-му процессу, тогда для остальных $(n-1)$ процессов распределению подлежат $\left(X-X_{i}\right)$ рабочих. Если $y_{i}\left(X_{i}\right)$ есть продукция, полученная от $i$-го процесса при выделении ему $x_{i}$ рабочих, то рекуррентное уравнение имеет вид:

$$
f_{n}(x)=y_{n}\left(x_{n}\right)+f_{n-1}\left(X-x_{n}\right)
$$

Очевидно, уравнение (10) удовлетворяет принципу оптимальности. В самом деле, какова бы ни была численность рабочих $x_{n}$ согласно определению метода оставшиеся $X-X_{n}$ рабочих распределяются оптимально.

Зная $f_{1}(x)=y_{1}(x)$ и имея рекуррентное соотношение (10), можно определить последовательность $\left\{f_{n}(x)\right\}$ доля любого значения $n$ и $X$.

\section{Общая схема решения состоит в следующем:}

Отрезок [OX] разбивается произвольными точками на части $\Delta ; 2 \Delta ;$ и т.д. и составляется расчетная таблица 3.

Максимизация производится методом прямого перебора вариантов, т.е. с помощью уравнения (10) определяется $f_{l}(x)$ при $n=1$ для значений $x=0, \Delta, 2 \Delta$. При найденной величине $f_{1}(x)$ определяется $f_{2}(x)$ для тех же значений $x$ и т.д.

Малоизученной проблемой является также решение задач нормирования труда методами математической статистики. Для эффективного решения данной задачи применим метод выборочного наблюдения.

Пример. На предприятии, по данным 10\% выборочного обследования выполнения норм выработки, среднее перевыполнение норм 25 рабочими составила $12 \%$, при среднеквадратическом отклонении 1,5\%. Найдем с вероятностью 0,954 доверительный интервал средней выполнимости норм выработки.
Здесь $T=25 ; \quad \tilde{x}=12 \% ; \quad \sigma_{b}=1.5 \% ; \quad F(t)=0.954 ;$ $t=2$.

Следовательно,

$$
\begin{aligned}
& \mu=\frac{15}{\sqrt{25}}=0,3 \% ; \quad \Delta=2 \cdot 0,3=0,6 \% . \\
& \Delta_{\%}=(0,6: 12) 100=5 \% .
\end{aligned}
$$

Построим следующий доверительный интервал: $12-5 \leq \widetilde{X} \leq 12+5$. Итак, с вероятностью 0,954 можно утверждать, что среднее перевыполнение норм выработки рабочими в день обследования находится в пределах от 7\% до $17 \%$.

Для определения необходимого объема выборочных наблюдений (количество рабочих или рабочих мест) методом фотографий, на наш взгляд, можно использовать приводимую ниже формулу:

$$
n=\frac{t^{2} \cdot \sigma^{2} \cdot N}{\Delta^{2} \cdot N+t^{2} \cdot \sigma^{2}},
$$

где $n$ - число выборочной совокупности; $N$ - численность генеральной совокупности; $t$ - коэффициент, который зависит от вероятности, с которой гарантируется заданная точность выборки; $\Delta^{2}$ - предельная ошибка выборки; $\sigma^{2}$ - дисперсия величины выборочной совокупности.

Пример.На заводе работает 100 токарей.Определим, сколько человек надо охватить наблюдением при нормировании труда. При этом принимаем $\varepsilon=0,3$, а $t=3$ (коэффициент $t$ находится по таблице). Ориентировочно принимаем, что из 100 токарей необходимо охватить (в первом приближении) наблюдением $\left(n_{l}\right)$. Исходные данные в таблице 4.

Используя данные таблицы, определим значения:

$$
\bar{Y}=\frac{\sum Y}{n}=\frac{22}{15}=1,47
$$


Таблица 4

\begin{tabular}{|l|l|l|l|}
$\begin{array}{l}\text { Кол-во } \\
\text { токарей }\end{array}$ & $\begin{array}{l}\text { Разброс величины потерь } \\
\text { времени в среднем за смену }(y), \\
\text { час }\end{array}$ & $Y-\bar{Y}$ & $\left(Y-\bar{Y}^{2}\right.$ \\
\hline 1 & 0,9 & 0,57 & 0,32 \\
\hline 2 & 2,0 & 0,53 & 0,28 \\
\hline 3 & 2,3 & 0,83 & 0,69 \\
\hline 4 & 1,1 & 0,37 & 0,14 \\
\hline 5 & 2,1 & 0,63 & 0,40 \\
\hline 6 & 1,6 & 0,13 & 0,017 \\
\hline 7 & 1,4 & 0,07 & 0,0049 \\
\hline 8 & 1,1 & 0,37 & 0,14 \\
\hline 9 & 2,2 & 0,73 & 0,53 \\
\hline 10 & 0,7 & 0,77 & 0,59 \\
\hline 11 & 0,8 & 0,67 & 0,45 \\
\hline 12 & 2,0 & 0,33 & 0,28 \\
\hline 13 & 1,8 & 0,33 & 0,11 \\
\hline 14 & 0,8 & 0,67 & 0,45 \\
\hline 15 & 1,2 & 0,27 & 0,073 \\
\hline & 22,0 & - & 4.475 \\
\hline
\end{tabular}

$\sigma^{2}=\frac{\sum(Y-\bar{Y})^{2}}{n_{1}}=\frac{4,475}{15}=0,30$

Подставляя значения $t$ и $\sigma^{2}$ в формулу получим:

$n_{2}=\frac{3^{2} \times 0.30 \times 100}{(0,3)^{2} \times 100+3^{2} \times 0,30}=\frac{270}{11,7}=23$ человека.

Следовательно, для обеспечения заданной точности необходимо обследовать не 15 токарей, а 23.

На предприятиях или в цехах с небольшой численностью рабочих величину внутрисменных потерь рабочего времени можно получить следующим методом. Для этого фотографии следует проводить выборочно в течение трех дней месяца (по одному дню в каждой декаде) с охватом 30-50\% рабочих разных специальностей, занятых соответственно в первой, второй и третьей сменах. Полученные данные распространяются на остальную часть рабочих и все рабочие дни месяца. Тогда возможная сумма внутрисменных потерь рабочего времени за месяц может быть рассчитана по формуле:

$$
t_{1}=t_{1}^{\prime} \cdot \frac{P_{0} \cdot m}{4}
$$

где $t_{1}$ - общая сумма потерь рабочего времени по той или иной причине за месяц, час;

$t_{1}^{\prime}$ - сумма потерь рабочего времени за дни обследования по охваченной группе рабочих по той же причине, час;

$P_{0}$ - общее число рабочих на объекте, человек;

$m$ - число рабочих дней в месяце; $n$ - число рабочих, охваченных фотографиями за все дни, человек.

Пример. $P_{0}=150$ человек, $m=21$ день, $n=70$ человек. Сумма потерь рабочего времени из-за недостатков в организации труда за дни обследования по группе охваченных рабочих $t_{1}^{\prime}=168$ час. Тогда общая сумма потерь рабочего времени по указанной причине в целом по цеху составить:

$$
t_{1}=168 \times \frac{150 \cdot 21}{70}=7560 \text { час. }
$$

Необходимую численность выборки можно определить так. Допустим, на предприятии решено уточнить нормы выработки на некоторой операции. Требуется определить, сколько должно быть проведено хронометражных наблюдений, чтобы ошибка при установлении новых норм выработки не превышала, скажем 5\%.

По предыдущим наблюдениям известно, что дисперсия фактической выборки $\sigma^{2}=16$ кг, а средняя выработка $\bar{x}=40$ кг. Необходимую численность наблюдений $n$ определяем по формуле:

$$
n=\frac{t^{2} \cdot \sigma^{2}}{\varepsilon^{2}}
$$

где $\varepsilon$ - размер допустимой ошибки выборки.

Так как ошибка в задаче задана в\%, формулу расчета запишем так:

$$
n=\frac{t^{2} \cdot \sigma^{2}}{\varepsilon^{2} \%}
$$


Таблица 5

\begin{tabular}{|l|l|l|l|l|l|}
\hline $\begin{array}{l}\text { Выполнение норм } \\
\text { выработки (\%) } x_{i}\end{array}$ & Число рабочих $f_{i}$ & $x_{i} \cdot f_{i}$ & $X_{i}-\bar{X}$ & $\left(x_{i}-\tilde{x}\right)^{2}$ & $\left(x_{i}-\tilde{x}\right)^{2} \cdot f_{i}$ \\
\hline 148 & 2 & 296 & 2,6 & 6,76 & 13,52 \\
\hline 150 & 4 & 600 & 0,6 & 0,36 & 1,44 \\
\hline 152 & 3 & 456 & 1,4 & 1,96 & 5,88 \\
\hline 154 & 1 & 154 & 3,4 & 11,56 & 11,56 \\
\hline Итого: & 10 & 1506 & $\mathrm{x}$ & $\mathrm{x}$ & 32,40 \\
\hline
\end{tabular}

где, $V \%$ - коэффициент вариации, исчисляемый по формуле

$$
V \%=\frac{\sigma}{\bar{X}} \cdot 100=\frac{\sqrt{16}}{40} \cdot 100=10 \%
$$

При вероятности 0,96 значение $t=1,96$ (по таблице), тогда необходимую численность выборки находим по формуле:

$$
n=\frac{t^{2} \cdot V^{2} \%}{\varepsilon^{2} \%} ; \quad n=\frac{1.96^{2} \cdot 10^{2}}{5^{2}}=15,3 .
$$

Следовательно, для уточнения норм выработки необходимо провести 15-16 хронометражных наблюдений.

Следует отметить, что при исследовании качества промышленной продукции, а также при установлении норм выработки часто пользуются малой выборкой.

Пример. Предположим, что на заводе с целью проверки выполнения норм выработки организована малая выборка (таблица 5).

В случайном бесповторном порядке отобрано 10

рабочих. Выборочная средняя $\widetilde{X}=1506: 10=150,6 \%$ . Выборочная дисперсия $\mu_{\text {м.b }}^{2}=32,4: 10=3,24$, стандартная ошибка

$$
\mu_{M . b}=\sqrt{\frac{3,24}{9}}=0,6 \%
$$

В условиях малой выборки при $k=n-1=9$ и $t=2,5$ с вероятностью $P_{k}|t|$, равной 0,966, предельная ошибка по абсолютной величине будет не больше $\Delta_{\text {M.b }}=2,50,6=1,5 \%$. Вероятность того, что это утверждение неправильно и ошибка может быть больше, чем 1,5\%, равна: $1-0,966=0,034$.

Доверительный интервал, в котором находится значение генеральной средней, можно определить как
$\widetilde{X} \pm 1,5 \%$, т.е. доверительный интервал будет выглядеть следующим образом: $150,6-1,5 \leq \bar{X} \leq 150,6+1,5$, где генеральная средняя лежит в интервале от $149,1 \%$ до $152,1 \%$.

Для изучения использования рабочего времени и работы оборудования используют моментные наблюдения. Особенностью моментного наблюдения является то, что по охвату объектов совокупности моментное наблюдение является сплошным, а по времени - выборочном. Сплошным оно является потому, что учету подлежат все единицы оборудования (или все рабочие). Выборочным оно является по той причине, что из всего фонда времени в наблюдение попадает лишь время, в течение которого ведется регистрация.

Для определения числа моментов наблюдения воспользуемся формулой

$$
n=\frac{t^{2} \sigma_{B}^{2}}{\Delta^{2}}
$$

Но так как все параметры этой формулы на стадии подготовки наблюдения неизвестны, то $\sigma_{B}^{2}$ целесообразно принять на уровне максимального значения дисперсии альтернативного признака, равного 0,25. Предельную ошибку $\Delta$ при таком расчете принято устанавливать в размере 5\% предполагаемого коэффициента потерь: $\Delta=0,5 \cdot 0,05=0,025$. Коэффициент доверия $t$ в практике подобных расчетов принимают равным 2, что гарантирует доверительный результат с вероятностью 0,954. При заданных условиях объем выборки (число станко - моментов) составит

$$
n=\frac{2^{2} \cdot 0,25}{(0,025)^{2}}=1600 .
$$

Предположим, что в результате проведенного моментного наблюдения коэффициент потерь времени в использовании оборудования составил 0,3 или $30 \%$. Предельную ошибку $\Delta$ можно определить так:

$$
\Delta=2 \sqrt{\frac{0,3 \cdot 0,7}{1600}}=0,02
$$


или 2\%. Доверительный интервал коэффициента потерь времени в генеральной совокупности составит: $30-2 \leq \bar{K}$ потерь\% $\leq 30+2$.
Это означает, что с вероятностью 0,954 можно утверждать, что потери времени оборудования не меньше $28 \%$ и не больше $32 \%$ [7, с. 115$]$.

\section{ЛИТЕРАТУРА}

1. Френкель А.А. Математические методы анализа динамики и прогнозирования производительности труда. М. Эко. 1972.

2. Четыркин Е.М. Статистические методы прогнозирования М., Статистика, 1977, 199 с.

3. Гойзман Э.И. Построение и анализ регрессионных зависимостей. М., 1979, 116 с.

4. Заварыкин В.М. Численные методы. М., Просвещение, 1991, 344 с.

5. Дж.Бигель. Управление производством. (Пер. с англ.).- М.: Мир, 1973, 301 с.

6. Балашевич В.А. Математические методы в управлении производством. - М.: ВШ, 1976, 292 с.

7. Ботащев Р.А., Байчорова С.К. Математические методы в задачах экономики.-Карачаевск, КЧГУ, 2018, — 220 с. ISBN978-5-8307-0538-7

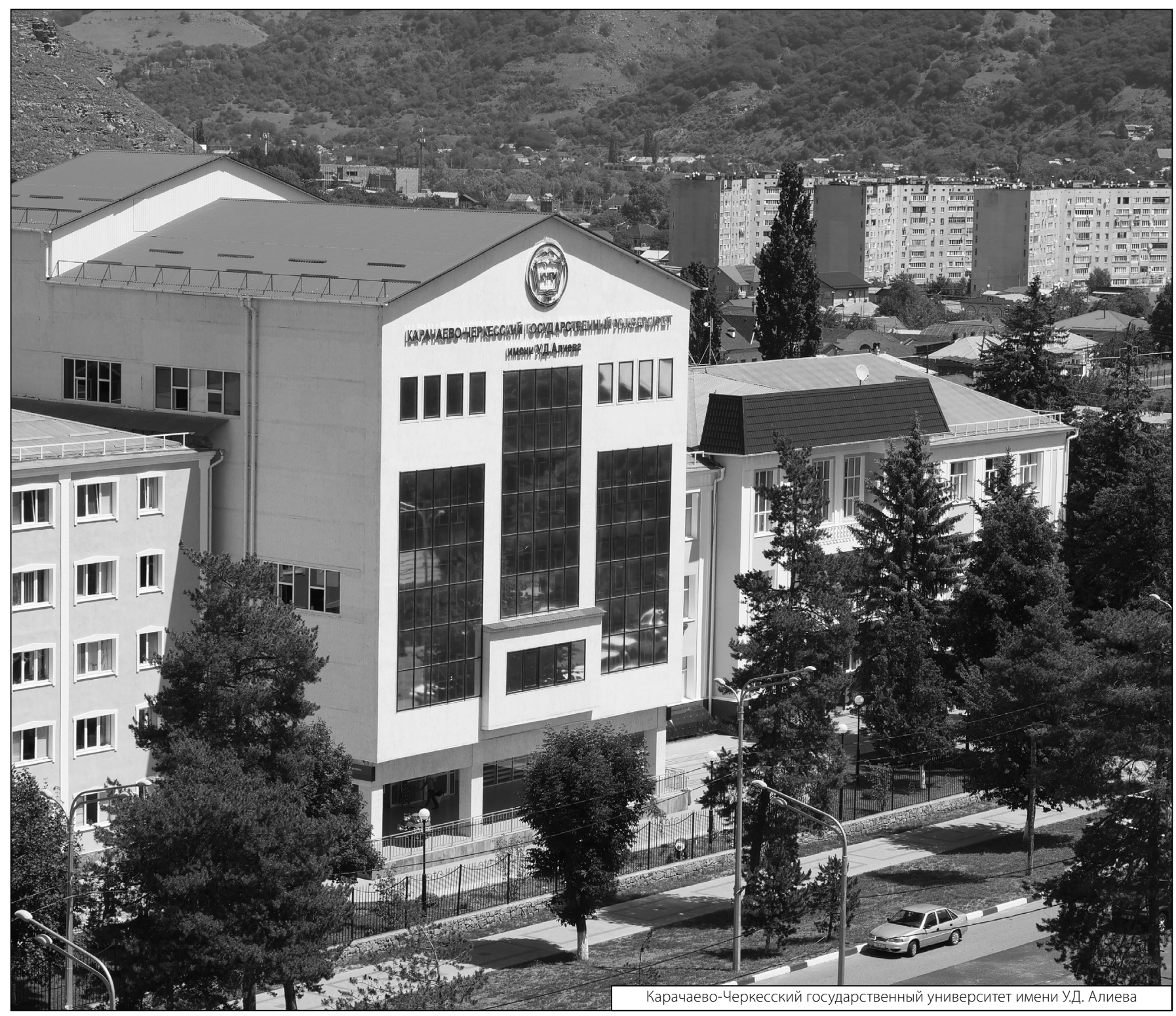

